\title{
Incidence and risk factors for extended post-operative length of stay following primary hip arthroplasty in a South African setting
}

\author{
Dlamini NF${ }^{1} \oplus$, Ryan $\mathrm{PV}^{2} \mathbb{\bullet}$, Moodley $\mathrm{Y}^{3} \oplus$
}

1 MBChB(UCT), MMed, FC Orth (SA); Consultant orthopaedic surgeon, Department of Orthopaedics, Prince Mshiyeni Memorial Hospital, Durban; and Department of Orthopaedics, Nelson R Mandela School of Medicine, University of KwaZulu-Natal, Durban, South Africa

$2 \mathrm{MBChB}(\mathrm{UCT})$, MMed, FC Orth (SA); Consultant orthopaedic surgeon, Department of Orthopaedics, Inkosi Albert Luthuli Central Hospital, Durban, South Africa

3 PhD; Research fellow, Department of Anaesthetics, Nelson R Mandela School of Medicine, University of KwaZulu-Natal, Durban, South Africa

Corresponding author: Dr NF Dlamini, Department of Orthopaedic Surgery, King Edward Hospital, Private BagX02, Congella 4013, South Africa; email: nkanyiso.dlamini@gmail.com; tel: +27723875344

\begin{abstract}
Background: This study sought to determine the incidence of extended post-operative length of stay (EPLoS) and its associated risk factors in South African primary hip arthroplasty patients.

Methods: This was a retrospective chart review study of 185 adults who underwent primary hip arthroplasty at a quaternary South African hospital. Data related to patient, clinical, and surgical characteristics were collected. Post-operative length of stay was calculated as the time (in days) between the dates of surgery and discharge from hospital. We defined EPLoS as any length of stay $\geq 75$ th percentile obtained for the entire study population. Data were analysed using univariate and multivariate statistical methods.

Results: The incidence of EPLoS was $28.1 \%$ (95\% confidence interval - Cl: 22.1-35.0\%). Risk factors for EPLoS included: female sex (odds ratio - OR: 4.63, 95\% Cl: 1.74-12.34; $p=0.002)$, patient's maximum walking distance <100 m (OR: 3.05, 95\% Cl: 1.05-8.89; $\mathrm{p}=0.041$ ) and extended duration of surgery (OR: 3.62, 95\% Cl: $1.31-10.01 ; \mathrm{p}=0.013$ ).
\end{abstract}

Conclusion: We provide a report of EPLoS and several associated risk factors in South African primary hip arthroplasty patients.

Level of evidence: Level 4

Key words: primary hip arthroplasty, primary hip replacement, South Africa, extended length of stay, risk factors

Citation: Dlamini NF, Ryan PV, Moodley Y. Incidence and risk factors for extended post-operative length of stay following primary hip arthroplasty in a South African setting. SA Orthop J 2019;18(1):40-46. http://dx.doi.org/10.17159/2309-8309/2019/v18n1a5

Editor: Dr C Snyckers, University of Pretoria, South Africa

Received: October 2017

Accepted: September 2018

Published: March 2019

Copyright: ( 2019 Dlamini NF. This is an open-access article distributed under the terms of the Creative Commons Attribution Licence, which permits unrestricted use, distribution and reproduction in any medium, provided the original author and source are credited.

Funding: None

Conflict of interest: All authors declare that there are no conflicts of interest regarding this research. 


\section{Introduction}

Increased global life expectancy has been linked to a higher burden of musculoskeletal conditions, including hip fracture and osteoarthritis. ${ }^{1}$ Untreated musculoskeletal conditions impact quality of life in afflicted patients and also have adverse consequences on healthcare expenditure and resource utilisation. ${ }^{1}$ These conditions would therefore have public health significance in resource-limited settings. Aside from non-communicable aetiologies, the global HIV epidemic has also been linked to the growing prevalence of orthopaedic disorders. ${ }^{2}$ Conservative medical therapy might not be effective in a large proportion of patients afflicted with orthopaedic hip conditions. Surgical intervention remains the only viable management option in these patients. ${ }^{3}$ The effectiveness of primary hip arthroplasty in reversing pain and loss of function associated with orthopaedic hip conditions is well described. ${ }^{3,4}$ Utilisation of primary hip arthroplasty as a surgical intervention for orthopaedic hip conditions has increased substantially over the past two to three decades, with this procedure now considered among the most common surgical procedures performed worldwide. ${ }^{4}$

A survey of orthopaedic surgeon members belonging to the South African Orthopaedic Association reported that each member in the country performed up to 43 hip arthroplasties each year. ${ }^{5}$ In addition, a lack of surgical expertise and other essential resources in surrounding countries has resulted in a number of patients from these countries being referred to South African hospitals for the procedure. ${ }^{6}$ In response to the increasing demand for primary hip arthroplasty, it is possible that many South African orthopaedic surgery units will in future adopt accelerated post-operative care pathways, in which the length of inpatient stay (and subsequent expenditure and resource utilisation for each patient) following surgery is reduced. ${ }^{7,8}$ An understanding of which patient, clinical, and surgical characteristics are associated with extended postoperative length of stay (EPLoS) in South African primary hip arthroplasty patients would have important future implications for the development of fast-track or accelerated surgical and recovery protocols implemented at orthopaedic surgery units in the country.

Therefore, the objectives of this study were to:

1. Determine the incidence of EPLoS in a sample of South African primary hip arthroplasty patients

2. Determine which patient, clinical, and surgical characteristics are associated with EPLoS in a sample of South African primary hip arthroplasty patients.

\section{Materials and methods}

\section{Study design, study setting, and study population}

This was a retrospective chart review study involving consecutive adult patients who were admitted for primary hip arthroplasty through a dedicated arthroplasty unit at a quaternary level hospital in KwaZulu-Natal, South Africa, between 23 September 2014 and 28 July 2016. Inclusion/exclusion criteria for this study are presented in Table I. Potential participants were identified from theatre lists during the specified study period.

\section{Data collection}

The medical records of all patients included in this study were reviewed and data related to various patient (demographics), clinical (comorbidities, presenting diagnosis, Thomas test with fixed flexion deformity [FFD], etc.), and surgical characteristics (nature of surgery, anaesthesia, surgical approach, duration of surgery,

Table I: Inclusion and exclusion criteria for the proposed study

\section{Inclusion criteria}

Patients aged 18 years or older

Patients who underwent primary hip arthroplasty at IALCH

between 23 September 2014 and 28 July 2016

Patient not previously included in study

and peri-operative blood transfusion) were collected using case report forms. We also collected data related to the occurrence of serious peri-operative complications, which we defined as a grade III or above peri-operative complication when using the Clavien-Dindo classification (includes: organ failure, critical care admission, re-operation, and mortality). ${ }^{9}$ Post-operative length of stay was calculated as the time (days) between the date of a patient's operation and the date that the patient was discharged from hospital.

The study outcome was EPLoS. This was defined as a postoperative length of stay $\geq 75$ th percentile calculated for the entire study population. This definition of EPLoS has been used in similar surgical studies conducted in overseas settings. ${ }^{10,11}$ Data were transferred from the case report forms to a Microsoft Excel ${ }^{\circledR}$ spreadsheet in preparation for analysis.

\section{Data analysis}

The median length of stay for the study population was calculated and is presented with an interquartile range. The incidence of EPLOS in this study was calculated using conventional epidemiological methods. ${ }^{12}$ The incidence of EPLoS in this study is presented as a percentage with $95 \%$ confidence intervals $(95 \% \mathrm{Cl})$. Potential associations between various patient, clinical, and surgical characteristics and EPLoS were investigated using univariate ( $X^{2}$ test, or Fisher's exact test) and multivariate (binary logistic regression) statistical methods. Results for the univariate statistical analysis are presented as frequencies and percentages. Characteristics with $p<0.100$ in the univariate analysis were selected for inclusion in the multivariate statistical analysis. This purposeful selection of variables for inclusion in the multivariate analysis was done to obtain the most parsimonious model possible. ${ }^{13}$ Model fit was assessed using a Hosmer-Lemeshow test. Results for the multivariable statistical analysis are presented as odds ratios (OR) with $95 \% \mathrm{Cl}$. A p-value of $<0.050$ was considered to be a statistically significant result. All statistical analyses were performed using the Statistical Package for the Social Sciences (SPSS) version 24.0 (IBM Corp, USA).

\section{Results}

\section{Derivation of study population and incidence of EPLOS}

The derivation of the study population and the incidence of EPLoS in this study is shown in Figure 1. Following the application of our study inclusion and exclusion criteria, our final study population consisted of 185 adult patients who underwent primary hip arthroplasty. The median post-operative length of stay for the study population was 5.0 days (interquartile range: $3.0-7.0$ days). The 75 th percentile for the study population post-operative length of 


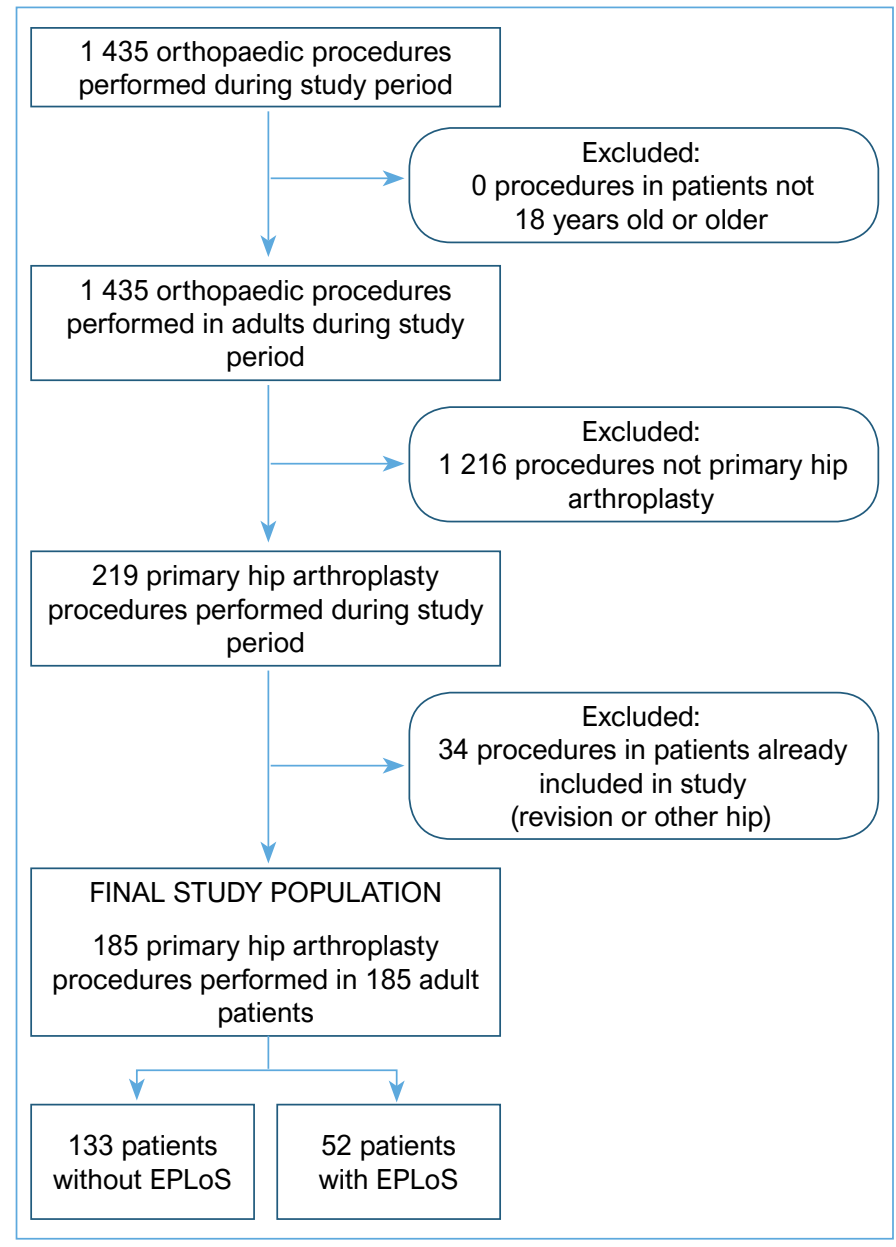

EPLoS: Extended post-operative length of stay

Figure 1. Study profile and incidence of EPLoS

stay was 7.0 days. A total of 52/185 patients experienced EPLoS following primary hip arthroplasty, with the calculated incidence of EPLoS being $28.1 \%$ (95\% Cl: $22.1-35.0 \%)$.

\section{Distribution of patient, clinical, and surgical characteristics in the study population}

The distribution of patient, clinical, and surgical characteristics in the study population is shown in Table II. A total of 43/185 patients were elderly (23.2\%). There was a higher proportion of female patients versus male patients in the study population ( $55.1 \%$ versus $44.9 \%$, respectively). With regard to comorbidity, $38.4 \%$ (71/185) of the study population were classified as having severe systemic disease (an American Society of Anesthesiologists score of $\geq 3$ ). The most prevalent comorbidities in the study population were hypertension ( $\mathrm{n} / \mathrm{N}=86 / 185,46.5 \%)$, obesity ( $\mathrm{n} / \mathrm{N}=84 / 185,45.4 \%$ ), and anaemia $(\mathrm{n} / \mathrm{N}=53 / 185,28.6 \%)$. Osteoarthritis was the most common presenting diagnosis ( $\mathrm{n} / \mathrm{N}=80 / 185,43.2 \%)$, followed by osteonecrosis $(\mathrm{n} / \mathrm{N}=61 / 185,33.0 \%)$ and other miscellaneous orthopaedic diagnoses $(\mathrm{n} / \mathrm{N}=44 / 185,23.8 \%)$. The most frequent miscellaneous diagnoses reported were hip dysplasia $(11 / 44$ patients $25.0 \%$ ), fracture (9/44 patients, $20.5 \%)$, ankylosis (8/44 patients $18.2 \%)$, and rheumatoid arthritis (7/44 patients, $15.9 \%$ ). Fixed flexion deformity (as per the Thomas test) was established for $138 / 185$ patients $(74.6 \%)$. Overall, $13.5 \%$ of the study population were classified as having severe hip deformation (FFD > 30 degrees). A total of $138 / 185$ patients (74.6\%) used an assistive device for mobilisation. Pain scores (visual analogue score - VAS) could only be established for $121 / 185$ patients (65.4\%). A total of $99 / 185$ patients had a VAS $\geq 7$ (53.5\%). We could only establish maximum walking distance for $58.4 \%(\mathrm{n} / \mathrm{N}=108 / 185)$ of the study population. A total of $52 / 185$ patients (28.1\%) could not walk $100 \mathrm{~m}$ or more. The median duration of surgery for the study population was 100.0 minutes (interquartile range: $75.0-125.0$ minutes). Urgent/ emergent surgical procedures were rare in the study population $(\mathrm{n} / \mathrm{N}=3 / 185,1.6 \%)$. Surgery was performed under general anaesthesia in $96 / 185$ patients (51.9\%). The standard posterior approach was used in $123 / 185$ procedures (66.5\%). Thirty-five patients $(18.9 \%)$ experienced surgery of extended duration, which we defined as a surgery with a duration $\geq 75$ th percentile calculated for the entire study population. Twenty-six patients in the study population required a peri-operative blood transfusion (14.1\%). Serious peri-operative complications were rare $(\mathrm{n} / \mathrm{N}=5 / 185,2.7 \%)$.

\section{Results of the univariate statistical analysis}

The results of the univariate statistical analysis are also shown in Table II. The proportions of several characteristics were statistically similar $(p \geq 0.050)$ between patients who experienced EPLoS and patients who did not experience EPLoS. These characteristics included: elderly age $(p=0.130)$, American Society of Anesthesiologists Score $(p=0.306)$, current smoker $(p=0.061)$, cardiovascular disease $(p=0.999)$, chronic obstructive pulmonary disease $(p=0.327)$, HIV $(p=0.764)$, diabetes $(p=0.999)$, anaemia $(p=0.970)$, obesity $(p=0.807)$, hypertension $(p=0.056)$, mobilisation with an assistive device $(p=0.937)$, VAS $(p=0.774)$, urgent/emergent surgery $(p=0.560)$, general anaesthesia $(p=0.739)$, and posterior surgical approach to the hip $(p=0.216)$. The proportions of the remaining characteristics were statistically different $(p<0.050)$ between patients who experienced EPLoS and patients who did not experience EPLoS. These characteristics included: sex $(p<0.001)$, presenting diagnosis $(p=0.011), F F D(p<0.011)$, patient's maximum walking distance $(p=0.009)$, extended duration of surgery $(p=0.003)$, peri-operative blood transfusion $(p<0.001)$, and serious perioperative complications $(p=0.023)$. We were unable to compute statistics for the characteristic 'renal impairment', as we found that no patients in our study population had this characteristic.

\section{Results of the multivariable statistical analysis}

The results of the multivariable statistical analysis are shown in Table III. Only nine of the characteristics investigated in the univariate analysis met the criteria of $p<0.100$ for inclusion in the multivariable analysis. These characteristics were sex, being a current smoker, hypertension, presenting diagnosis, FFD, patient's maximum walking distance, extended duration of surgery, peri-operative blood transfusion, and serious peri-operative complications. Of these characteristics, only three were found to be independently associated with EPLoS. These characteristics were female sex (when compared with males, OR: $4.63,95 \%$ $\mathrm{Cl}$ : 1.74-12.34; $p=0.002$ ), patient's maximum walking distance $<100 \mathrm{~m}$ (when compared with the reference of walking distance $\geq 100$ m, OR: 3.05, 95\% Cl:1.05-8.89; $p=0.041$ ), and extended duration of surgery (when compared with surgery duration $<75$ th percentile obtained for the entire study population, OR: $3.62,95 \%$ $\mathrm{Cl}: 1.31-10.01 ; p=0.013)$. The result for the Hosmer-Lemeshow test indicated adequate model fit $(p>0.050)$.

\section{Discussion}

The median post-operative length of stay in our South African study population was much shorter than that reported for British, American, and Pakistani patient populations undergoing primary hip arthroplasty (median of five days in our study population 
Table II: Distribution of patient/clinical characteristics in the study population and results of the univariate statistical analysis*

\begin{tabular}{|c|c|c|c|c|c|}
\hline $\begin{array}{c}\text { Clinical } \\
\text { characteristic }\end{array}$ & Sub-category & All patients $(\mathrm{N}=185)$ & No EPLoS $(n=133)$ & EPLoS (n=52) & p-value \\
\hline \multirow[t]{3}{*}{ Age $>65$ years old } & & & & & 0.130 \\
\hline & Yes & $43(23.2)$ & $27(20.3)$ & $16(30.8)$ & \\
\hline & No & $142(76.8)$ & $106(79.7)$ & $36(69.2)$ & \\
\hline \multirow[t]{3}{*}{ Sex } & & & & & $<0.001$ \\
\hline & Female & $102(55.1)$ & $60(45.1)$ & $42(80.8)$ & \\
\hline & Male & $83(44.9)$ & $73(54.9)$ & $10(19.2)$ & \\
\hline \multirow[t]{3}{*}{ ASA score $\geq 3$} & & & & & 0.306 \\
\hline & Yes & $71(38.4)$ & $48(36.1)$ & $23(44.2)$ & \\
\hline & No & $114(61.6)$ & 85 (63.9) & $29(55.8)$ & \\
\hline \multirow[t]{3}{*}{ Current smoker } & & & & & 0.061 \\
\hline & Yes & $42(22.7)$ & $35(26.3)$ & $7(13.5)$ & \\
\hline & No & $143(77.3)$ & $98(73.7)$ & $45(86.5)$ & \\
\hline \multirow{3}{*}{$\begin{array}{l}\text { Cardiovascular } \\
\text { disease }\end{array}$} & & & & & 0.999 \\
\hline & Yes & $10(5.4)$ & $7(5.3)$ & $3(5.8)$ & \\
\hline & No & $175(94.6)$ & $126(94.7)$ & $49(94.2)$ & \\
\hline \multirow[t]{3}{*}{ COPD } & & & & & 0.327 \\
\hline & Yes & $21(11.4)$ & $17(12.8)$ & $4(7.7)$ & \\
\hline & No & $164(88.6)$ & $116(87.2)$ & $48(92.3)$ & \\
\hline \multirow[t]{3}{*}{ HIV } & & & & & 0.764 \\
\hline & Yes & $40(21.6)$ & 105 (78.9) & $40(76.9)$ & \\
\hline & No & $145(78.4)$ & $28(21.1)$ & $12(23.1)$ & \\
\hline \multirow[t]{3}{*}{ Diabetes } & & & & & 0.999 \\
\hline & Yes & $17(9.2)$ & $12(9.0)$ & $5(9.6)$ & \\
\hline & No & $168(90.8)$ & $121(91.0)$ & $47(90.4)$ & \\
\hline \multirow[t]{3}{*}{ Renal impairment } & & & & & UC \\
\hline & Yes & $0(0.0)$ & $0(0.0)$ & $0(0.0)$ & \\
\hline & No & $185(100.0)$ & $133(100.0)$ & $52(100.0)$ & \\
\hline \multirow[t]{3}{*}{ Anaemia } & & & & & 0.970 \\
\hline & Yes & $53(28.6)$ & $38(28.6)$ & $15(28.8)$ & \\
\hline & No & $132(71.4)$ & 95 (71.4) & 37 (71.2) & \\
\hline \multirow[t]{4}{*}{ Obesity } & & & & & 0.807 \\
\hline & CNBE & $11(5.9)$ & $7(5.3)$ & $4(7.7)$ & \\
\hline & Yes & $84(45.5)$ & $61(45.8)$ & $23(44.2)$ & \\
\hline & No & $90(48.6)$ & $65(48.9)$ & $25(48.1)$ & \\
\hline \multirow[t]{3}{*}{ Hypertension } & & & & & 0.056 \\
\hline & Yes & $86(46.5)$ & $56(42.1)$ & $30(57.7)$ & \\
\hline & No & $99(53.5)$ & $77(57.9)$ & $22(42.3)$ & \\
\hline \multirow[t]{4}{*}{ Presenting diagnosis } & & & & & 0.011 \\
\hline & Other & $44(23.8)$ & $25(18.8)$ & $19(36.6)$ & \\
\hline & Osteonecrosis & $61(33.0)$ & $51(38.3)$ & $10(19.2)$ & \\
\hline & Osteoarthritis & $80(43.2)$ & $57(42.9)$ & $23(44.2)$ & \\
\hline \multirow[t]{4}{*}{ FFD >30 degrees } & & & & & 0.011 \\
\hline & CNBE & $47(25.4)$ & $26(19.6)$ & $21(40.3)$ & \\
\hline & Yes & $25(13.5)$ & $18(13.5)$ & $7(13.5)$ & \\
\hline & No & $113(61.1)$ & $89(66.9)$ & $24(46.2)$ & \\
\hline \multirow{3}{*}{$\begin{array}{l}\text { Mobilises with } \\
\text { assistive device }\end{array}$} & & & & & 0.937 \\
\hline & Yes & $138(74.6)$ & $99(74.4)$ & $39(75.0)$ & \\
\hline & No & $47(25.4)$ & $34(25.6)$ & $13(25.0)$ & \\
\hline \multirow[t]{4}{*}{ VAS $\geq 7$} & & & & & 0.774 \\
\hline & CNBE & $63(34.1)$ & $47(35.3)$ & $16(30.8)$ & \\
\hline & Yes & $99(53.5)$ & 69 (51.9) & $30(57.7)$ & \\
\hline & No & $22(12.4)$ & $17(12.8)$ & $6(11.5)$ & \\
\hline
\end{tabular}


Table II continued from page 43

Walking distance $<100 \mathrm{~m}$

\section{$<100 \mathrm{~m}$}

\begin{tabular}{|l|l|}
\hline CNBE & $77(41.6)$ \\
\hline Yes & $52(28.1)$ \\
\hline No & $56(30.3)$ \\
\hline
\end{tabular}

\section{Urgent/emergent \\ surgery}

$56(30.3)$

$3(1.6)$
$182(98.4)$

96 (51.9)

89 (48.1)
0.009

\begin{tabular}{|l|l|}
\hline $59(44.4)$ & $18(34.6)$ \\
\hline $29(21.8)$ & $23(44.2)$ \\
\hline $45(33.8)$ & $11(21.2)$
\end{tabular}

11 (21.2)

0.560

\begin{tabular}{|l|l|}
\hline $3(2.3)$ & $0(0.0)$ \\
\hline $130(97.7)$ & $52(100.0)$ \\
\hline
\end{tabular}

0.739

\begin{tabular}{|l|l|}
\hline $68(51.1)$ & $28(53.8)$ \\
\hline $65(48.9)$ & $24(46.2)$ \\
\hline
\end{tabular}

0.216

\begin{tabular}{|c|c|c|c|}
\hline Yes & $123(66.5)$ & $92(69.2)$ & $31(59.6)$ \\
\hline No & 62 (33.5) & $41(30.8)$ & $21(40.4)$ \\
\hline
\end{tabular}

0.003

17 (32.7)

35 (67.3)

$115(86.5)$

$<0.001$

16 (30.8)

$36(69.2)$

0.023

\begin{tabular}{|l|l|l|}
\hline $5(2.7)$ & $1(0.8)$ & $4(7.7)$ \\
\hline $180(97.3)$ & $132(99.2)$ & $48(92.3)$ \\
\hline
\end{tabular}

$26(14.1)$

$159(85.8)$

$10(7.5)$

$123(92.5)$

Yes
No

180 (97.3)

$132(99.2)$
$48(92.3)$

Peri-operative

${ }^{*}$ Results expressed as frequencies (\%).

$p<0.050$ was considered a statistically significant result

EPLOS: extended post-operative length of stay; ASA: American Society of Anesthesiologists; COPD: chronic obstructive pulmonary disease; UC: unable to compute; CNBE: could not be established; FFD: fixed flexion deformity; VAS: visual analogue score

Table III: Results of the multivariate statistical analysis*

\begin{tabular}{|c|c|c|c|}
\hline Clinical characteristic & Sub-category & OR $(95 \% \mathrm{CI})$ & p-value \\
\hline \multirow[t]{2}{*}{ Sex } & Female & $4.63(1.74-12.34)$ & 0.002 \\
\hline & Male & Reference & - \\
\hline \multirow[t]{2}{*}{ Current smoker } & Yes & $1.15(0.36-3.66)$ & 0.817 \\
\hline & No & Reference & - \\
\hline \multirow[t]{2}{*}{ Hypertension } & Yes & $1.36(0.59-3.11)$ & 0.470 \\
\hline & No & Reference & - \\
\hline \multirow[t]{3}{*}{ Presenting diagnosis } & Other & $2.25(0.83-6.13)$ & 0.113 \\
\hline & Osteonecrosis & $0.70(0.24-2.01)$ & 0.507 \\
\hline & Osteoarthritis & Reference & - \\
\hline \multirow[t]{3}{*}{ FFD $>30$ degrees } & CNBE & $4.80(1.72-13.34)$ & $\mathrm{n} / \mathrm{a}$ \\
\hline & Yes & $0.52(0.14-1.91)$ & 0.326 \\
\hline & No & Reference & - \\
\hline \multirow[t]{3}{*}{ Walking distance $<100 \mathrm{~m}$} & CNBE & $0.48(0.15-1.53)$ & $\mathrm{n} / \mathrm{a}$ \\
\hline & Yes & $3.05(1.05-8.89)$ & 0.041 \\
\hline & No & Reference & - \\
\hline \multirow[t]{2}{*}{ Extended duration of surgery } & Yes & $3.62(1.31-10.01)$ & 0.013 \\
\hline & No & Reference & - \\
\hline \multirow[t]{2}{*}{ Peri-operative blood transfusion } & Yes & $2.35(0.80-6.88)$ & 0.120 \\
\hline & No & Reference & - \\
\hline \multirow[t]{2}{*}{ Peri-operative complication } & Yes & $11.77(0.95-145.54)$ & 0.055 \\
\hline & No & Reference & - \\
\hline
\end{tabular}

${ }^{*}$ Results adjusted for confounders. Only characteristics with $p<0.100$ in the univariate statistical analysis included in the multivariable statistical analysis.

$p<0.050$ was considered a statistically significant result.

OR: odds ratio; Cl: confidence interval; FFD: fixed flexion deformity; CNBE: could not be established 
versus seven to eight days in the other primary hip arthroplasty populations). ${ }^{10,14-16}$ South Africa is severely impacted by high levels of non-communicable disease, ${ }^{17}$ trauma/injury, ${ }^{18}$ and HIV infection, ${ }^{19}$ all of which are associated with the development of musculoskeletal/orthopaedic disease. ${ }^{20-23}$ This has resulted in a growing demand for hip arthroplasty in the country. ${ }^{6}$ However there are staffing and economic challenges in running orthopaedic surgical units in the public sector, ${ }^{24}$ and the availability of beds in these public hospitals might also be a concern. ${ }^{25}$ In order to cope with the higher demand for hip arthroplasty, some hospitals are beginning to implement fast-track protocols which are aimed at reducing post-operative length of stay while minimising the rate of post-discharge complications in suitable patients who undergo the surgical procedure. ${ }^{26}$ This might possibly explain the difference in median post-operative length of stay following primary hip arthroplasty between South African and overseas populations. ${ }^{10,14-16}$ With regard to EPLoS following hip arthroplasty, the literature is scant. However, there is one American study which reported EPLoS in this surgical population. ${ }^{10}$ In that study, the 75 th percentile for the population post-operative length of stay was 14.0 days, which is twice that reported for our study. ${ }^{10}$ Furthermore, one-third of the American study population experienced EPLoS. ${ }^{10}$ As with our findings for median post-operative length of stay, the discrepancy in EPLoS between the American study population and our South African study population must be viewed in the context of a growing demand for hip arthroplasty in South Africa and the disproportionate availability of healthcare resources between South African and American settings. ${ }^{27}$

We found statistically significant univariate associations between several characteristics (including: sex, presenting diagnosis, FFD, patient's maximum walking distance, extended duration of surgery, peri-operative blood transfusion, and serious post-operative complications). These findings are not unique to our study. Other overseas studies have reported univariate statistical associations between these/similar characteristics and post-operative length of stay in hip arthroplasty patients. ${ }^{14,16}$ We found three characteristics to be independently associated with EPLoS (including: sex, patient's maximum walking distance and extended duration of surgery). Female sex was found to be associated with an almost five-fold increase in the risk of experiencing EPLoS following primary hip arthroplasty. Abbas et al., reported an almost two-fold increase in the risk of EPLoS for women undergoing hip arthroplasty in a Pakistani setting..$^{16}$ Dall et al., also reported a multivariate statistical association (without describing the magnitude of risk) between female sex and longer post-operative length of stay a British hip arthroplasty population. ${ }^{14}$ Therefore, our findings for female sex appear, in general, to be in agreement with the published literature. However, the difference in the magnitude of odds ratios for female sex obtained in our study and the study of Abbas et al. ${ }^{16}$ requires further investigation. The characteristics of patient's maximum walking distance have not been specifically investigated as potential risk factors for EPLoS following hip arthroplasty in the published literature. However, these characteristics are components of the pre-operative Harris Hip Score, ${ }^{28}$ which has been shown by Dall et al. ${ }^{14}$ to be associated with length of stay following hip arthroplasty. Specifically, these characteristics appear to be associated with mobility and functional status in patients with hip conditions. ${ }^{28}$ Therefore, our findings highlight the potential importance of preoperative functional status and ambulation on the post-operative recovery period in South African primary hip arthroplasty patients. Lastly, we found extended duration of surgery to be associated with an almost four-fold higher risk of experiencing EPLoS. This is somewhat in agreement with the British study of Foote et al., who also report extended duration of surgery to be independently associated with a higher risk of EPLoS. ${ }^{15}$ However, as with the patients' sex, there appears to be a difference in the magnitude of odds ratios for surgery duration between our study and the study of Foote and colleagues. ${ }^{15}$ Attempts to should be made to reduce the duration of hip arthroplasty in our setting, possibly through the application of benchmarks and optimisation of surgical technique.

The risk factors identified in our study can be incorporated into future risk stratification systems for EPLoS in South African orthopaedic units. Similar risk stratification systems based on identified risk factors for EPLoS following primary hip arthroplasty have been proposed by Abbas et al., ${ }^{16}$ and Foote et al. ${ }^{15}$ These risk stratification systems are required to be developed and validated for performance in a separate surgical cohort. ${ }^{29}$ This step is beyond the scope of the dataset used in our study and requires further research.

There were several characteristics which were not found to be associated with EPLoS during the univariate statistical analysis, or following inclusion in the multivariable statistical analysis. There are two explanations for the lack of statistical association between these characteristics and EPLoS in our study. First, it might be possible that these characteristics, while identified as risk factors in overseas settings, are genuinely not associated with EPLOS in South African hip arthroplasty patients. Discordance in clinical risk factors between overseas/South African surgical populations and other post-operative outcomes has been described elsewhere. ${ }^{30}$ It might be worthwhile to involve overseas collaborators with access to overseas patient data in future research such that valid comparisons of risk factors between our settings can be made. Secondly, it is possible that a larger sample size than 185 patients would be required to investigate the impact of these characteristics on EPLoS. A potential solution to this would be a collaborative study involving as many hospitals which offer orthopaedic surgical services as possible.

Our study had several strengths. Our study is, to the best of our knowledge, the only South African study which specifically investigates EPLoS following primary hip arthroplasty. Another strength of our study is that we included data on HIV infection in our statistical analyses. This is important as the prevalence of HIV is usually much lower in American and British populations, ${ }^{19}$ and so our study provides important information on the impact of this characteristic in settings with a high burden of HIV infection. The final strength of our study is that while our sample size appeared modest, it still allowed for us to perform a multivariable statistical analysis to determine independent risk factors for EPLoS without any serious violation of statistical rules of thumb. ${ }^{31}$ Our study also had several limitations. First, as this study was conducted at a single, dedicated arthroplasty unit in a quaternary level hospital with standardised pre- and post-operative protocols in place, it might be argued that our study findings lack generalisability. As for our solution for the challenge related to the lack of statistical association between several characteristics and EPLOS, we recommend that collaborative studies involving hospitals at various levels of service delivery are conducted to determine the generalisability of our study findings. In addition, we were unable to investigate the impact of the Harris Hip Score in our study due to poor documentation of this characteristic in the patient medical records. We did, however, find that components of the Harris Hip Score were statistically associated with EPLoS, and it is therefore possible that the composite Harris Hip Score might also be associated with EPLoS. Prospective research wherein data collection for the Harris Hip Score is standardised is required. Finally, we did not report the impact of EPLoS on healthcare expenditure or post-discharge complications. These outcomes can only be appropriately investigated through the conduct of prospective research studies. 
In conclusion, we found several risk factors for EPLoS following primary hip arthroplasty in South African patients. These risk factors included sex, patient's maximum walking distance, and extended duration of surgery. Further research is required to confirm our study findings, as well as address the limitations identified in our study.

\section{Ethics statement}

This study was approved by the University of KwaZulu-Natal Biomedical Research Ethics Committee (Protocol: BE526/17). No benefits of any form have been received from a commercial party related directly or indirectly to the subject of this article.

\section{Declarations}

The authors declare authorship of this article and that they have followed sound scientific research practice. This research is original and does not transgress plagiarism policies.

\section{Author contributions}

This study formed part of the postgraduate medical studies of NFD. NFD conceptualised the research idea, executed the research protocol, and wrote the manuscript. PVR was involved in the conceptualisation of the research idea and provided a critical review of the manuscript. YM was involved in the conceptualisation of the research idea, performed the statistical analysis, and provided a critical review of the manuscript.

\section{ORCID}

Dlamini NF (iD http://orcid.org/0000-0003-2154-0199

Ryan PV ii http://orcid.org/0000-0002-0957-6482

Moodley Y (D) http://orcid.org/0000-0002-4119-1734

\section{References}

1. Woolf AD, Pfleger B. Burden of major musculoskeletal conditions. Bull World Health Organ 2003;81:646-56.

2. Lima AL, Godoy AL, Oliveira PR, et al. Orthopedic complications in HIV patients. Rev Bras Ortop 2009;44:186-90.

3. Singh JA. Epidemiology of knee and hip arthroplasty: a systematic review. Open Orthop J 2011;5:80-85.

4. Merx $\mathrm{H}$, Dreinhofer $\mathrm{K}$, Schrader $\mathrm{P}$, et al. International variation in hip replacement rates. Ann Rheum Dis 2003;62:222-26.

5. van Zyl AA, van der Merwe JF, Steyn R. Epidemiological study of total hip replacement in South Africa. Orthopaedic Proceedings 2005;87-B:278-78.

6. Lisenda L, Mokete K, Nwokeyi Y, Gureja YP, Lukhele M Epidemiological study of total hip replacement in South Africa. Acta Orthop Belg 82:2016.

7. Stambough JB, Nunley RM, Curry MC, Steger-May K, Clohisy JC Rapid recovery protocols for primary total hip arthroplasty can safely reduce length of stay without increasing readmissions. $J$ Arthroplasty 2015;30:521-26.

8. Wilches C, Sulbaran JD, Fernandez JE, Gisbert JM, Bausili JM Pelfort $X$. Fast-track recovery technique applied to primary total hip and knee replacement surgery. Analysis of costs and complications. Rev Esp Cir Ortop Traumatol 2017;61:111-16.

9. Dindo D, Demartines N, Clavien PA. Classification of surgical complications: a new proposal with evaluation in a cohort of 6336 patients and results of a survey. Ann Surg 2004;240:205-13.

10. Collins TC, Daley J, Henderson WH, Khuri SF. Risk factors for prolonged length of stay after major elective surgery. Ann Surg 1999;230:251-59.

11. Bou Monsef J, Boettner F. Blood management may have an impact on length of stay after total hip arthroplasty. HSS J 2014;10:124-30.

12. Crichton $\mathrm{N}$. Information point: prevalence and incidence. J Clin Nurs 2000;9:188.

13. Bursac Z, Gauss CH, Williams DK, Hosmer DW. Purposefu selection of variables in logistic regression. Source Code Biol Med 2008;3:17.

14. Dall GF, Ohly NE, Ballantyne JA, Brenkel IJ. The influence of pre-operative factors on the length of in-patient stay following primary total hip replacement for osteoarthritis: a multivariate analysis of 2302 patients. J Bone Joint Surg Br 2009;91:434-40.
15. Foote J, Panchoo K, Blair P, Bannister G. Length of stay following primary total hip replacement. Ann $R$ Coll Surg Engl 2009;91:500-504.

16. Abbas K, Umer M, Qadir I, Zaheer J, ur Rashid H. Predictors of length of hospital stay after total hip replacement. J Orthop Surg (Hong Kong) 2011;19:284-87.

17. Mayosi BM, Flisher AJ, Lalloo UG, Sitas F, Tollman SM, Bradshaw D. The burden of non-communicable diseases in South Africa. Lancet 2009;374:934-47.

18. Saggie J. Trauma: South Africa's other epidemic. S Afr Med J 2013;103:589-90.

19. Wang $\mathrm{H}$, Wolock TM, Carter A, et al. Estimates of global, regional, and national incidence, prevalence, and mortality of HIV, 1980-2015: the Global Burden of Disease Study 2015. Lancet HIV 2016;3:e361-87.

20. Oliver JE, Silman AJ. Risk factors for the development of rheumatoid arthritis. Scand J Rheumatol 2006;35:169-74.

21. Shah KN, Racine J, Jones LC, Aaron RK. Pathophysiology and risk factors for osteonecrosis. Curr Rev Musculoskelet Med 2015;8:201-209.

22. Kole AK, Roy R, Kole DC. Musculoskeletal and rheumatological disorders in HIV infection: Experience in a tertiary referral center. Indian J Sex Transm Dis 2013;34:107-12.

23. Noordin S, Wright JG, Howard AW. Global relevance of literature on trauma. Clin Orthop Relat Res 2008;466:2422-27.

24. Govender S. Basic research in orthopedics: South Africa. Indian J Orthop 2009;43:324-25.

25. Mac Mahon AG. Non-emergency patient transport - an integral part of accessible comprehensive health care. S Afr Med J 2011;101:684; 686.

26. Riemer B, Maclntyre K, Nortje M, Dower B, Grobler G, Springfield M. Rapid mobilisation following total hip and knee arthroplasty. $S A$ Orthop J 2017;16:58-61.

27. Benatar SR. Health care reform in the new South Africa. $N$ Engl J Med 1997;336:891-95.

28. McLean JM, Cappelletto J, Clarnette J, et al. Normal population reference values for the Oxford and Harris Hip Scores - electronic data collection and its implications for clinical practice. Hip Int 2016.

29. Moodley Y, Naidoo P, Biccard BM. The South African Vascular Surgical Cardiac Risk Index (SAVS-CRI): a prospective observational study. S Afr Med J 2013;103:746-50.

30. Moodley Y, Biccard BM. Predictors of in-hospital mortality following non-cardiac surgery: Findings from an analysis of a South African hospital administrative database. S Afr Med J 2015;105:126-29.

31. Vittinghoff E, McCulloch CE. Relaxing the rule of ten events per variable in logistic and Cox regression. Am J Epidemiol 2007;165:710-18. 